\title{
Real-time areal precipitation determination from radar by means of statistical objective analysis
}

\author{
E. -M. Gerstner ${ }^{a}$, G. Heinemann ${ }^{b, *}$ \\ ${ }^{a}$ Meteorological Institute, University of Bonn, Auf dem Hügel 20, 53121 Bonn, Germany \\ b Department of Environmental Meteorolology, University of Trier, 54286 Trier, Germany
}

Received 19 January 2006; received in revised form 21 December 2007; accepted 16 January 2008

\section{KEYWORDS \\ Radar precipitation; \\ Statistical objective analysis}

\begin{abstract}
Summary Precipitation measurement by radar allows for areal rainfall determination with a high spatial and temporal resolution. However, hydrological applications require an accuracy of the precipitation quantification which cannot be obtained by today's weather radar devices. The quality of the radar-derived precipitation can be significantly improved with the aid of ground measurements. In this paper, a complete processing pipeline for real-time radar precipitation determination using a modified statistical objective analysis method is presented. Thereby, several additional algorithms, such as a dynamical use of $Z-R$ relationships, a bias correction and an advection correction scheme are employed. The performance of the algorithms is tested for several case studies. For an error analysis, an eight months data set of X-band radar scans and rain gauge precipitation measurements is used. We show a reduction in the radar-rain gauge RMS difference of up to $59 \%$ for the optimal combination of the different algorithms.

(C) 2008 Elsevier B.V. All rights reserved.
\end{abstract}

\section{Introduction}

Areal precipitation is the most important input quantity for hydrological applications. For precipitation measurements, weather radar with a high spatial and temporal resolution is widely used. The success of hydrological simulations, however, is highly dependent on an accurate precipitation quantification, which is still a challenge for today's radar systems. Particularly, the patterns associated with summertime con-

\footnotetext{
"Corresponding author.

E-mail addresses: eva-maria.gerstner@uni-bonn.de (E.-M. Gerstner), heinemann@uni-trier.de (G. Heinemann).
}

vective strong rain events in mid-latitudes are neither captured well by simulations of current weather forecast models nor by the relatively sparse operational rain gauge networks of the national weather services. Numerous error sources reduce the accuracy of the measurement, e.g., the attenuation of the radar beam by heavy rainfall, ground clutter, beam occlusion or the choice of the radar reflectivityrain rate $(Z-R)$ relationship. The latter depends on the drop size distribution, which is unknown in general, but is variable for different precipitation events. It is also variable for the same type of precipitation (e.g. for different stages of convective cloud development, Reudenbach et al., 2001). Therefore, precipitation measurement based only on 
weather radar data cannot replace in situ rain gauge measurements (Joss and Waldvogel, 1990). Rain gauges, on the other hand, are representative only in a small neighbourhood of their location and, thus, also cannot achieve the desirable accuracy in areal precipitation determination. Several recent approaches, however, show that radar precipitation measurements can lead to reasonable results in synergy with rain gauge measurements of the Next Generation Weather Radar (NEXRAD) Program (Matsoukas et al., 1999; Fulton et al., 1998; Fulton, 1999; Pereira Fo and Crawford, 1999; Stellman et al., 2001). This is also the basis for the operational approach at the German Meteorological Service called RADOLAN (radar online calibration using rain gauges (DWD, 2004)).

The aim of this study is to improve the quality of the radar precipitation by a statistical objective analysis (SOA) method, which adjusts the radar data pixel by pixel with the help of the rain gauge measurements. Hereby, special focus lies on real-time areal precipitation quantification, e.g., for flood forecasting. A complete processing pipeline for real-time areal radar precipitation determination is presented. Therein, a series of algorithms are employed, e.g., an automatic determination method of $Z-R$ relationships for convective and stratiform precipitation events, an advection correction and a real-time statistical objective analysis. The parameters of the procedures are optimized and adjusted to the X-band radar of the Meteorological Institute of the University of Bonn (MIUB). In the present paper we first present a complete processing pipeline for realtime radar precipitation determination and several tests for case studies in order to demonstrate the effects of the different algorithm components. Then an error study is performed for the different components of the algorithm and their interplay is shown for two summer seasons (1998 and 1999) comprising eight months of data.

\section{Data basis}

The observation area is limited by the range of the MIUB radar (latitude: $50^{\circ} 43^{\prime} 53.5^{\prime \prime}$, longitude: $07^{\circ} 04^{\prime} 31.7^{\prime \prime}$, height: 98.5 $\mathrm{m})$, which measures within a maximum radius of $100 \mathrm{~km}$, of which only the first $50 \mathrm{~km}$ are analyzed (Fig. 1). The MIUB operates a pulse radar of the type Selenia METEOR-200 with a wavelength of $3.2 \mathrm{~cm}$ (X-band). The radial resolution of the raw data is $250 \mathrm{~m}$. For precipitation quantification, azimuth scans with an elevation of $2.5^{\circ}$ are used. The temporal resolution is $15 \mathrm{~min}$ for 1998 and $5-10 \mathrm{~min}$ for 1999 . A vertical reflectivity profile has not been available for the study. Beam occlusion effects occur in a small sector south of the radar.

For ground precipitation measurement, 21 rain gauges (Pluvio and Thies-Clima) with a temporal resolution of 5 min ( 15 operated by MIUB and 6 operated by the local authorities of the 'Erftverband') as well as 21 rain gauges with a temporal resolution of $24 \mathrm{~h}$ (daily measurements, operated by the German Meteorological Service (DWD)) are used. The spatial distribution of the rain gauges is shown in Fig. 2. They form two clusters: the cluster with the highresolution rain gauges (cluster I) is located mainly in the southwest, while the cluster with the daily resolution rain gauges (cluster II) is located mainly in the northeast of the radar area. Within these clusters the rain gauges show a relatively uniform distribution. The mean distance of the rain gauges does not exceed $6 \mathrm{~km}$. The summer periods investigated in the present paper have been part of intensive observation periods of the collaborative research centre

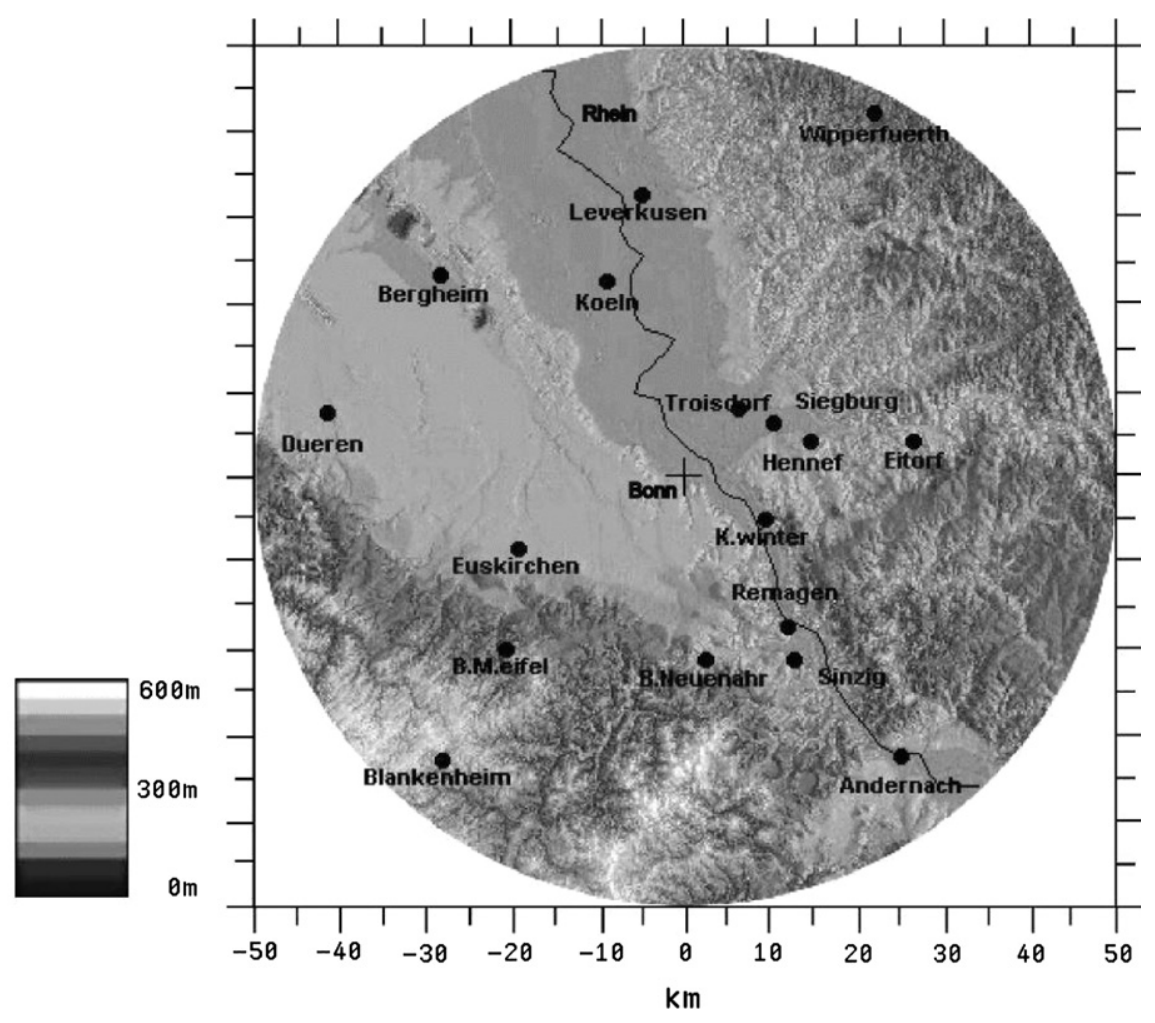

Figure 1 Observation area with orography (shaded). The city of Bonn is in the centre. 

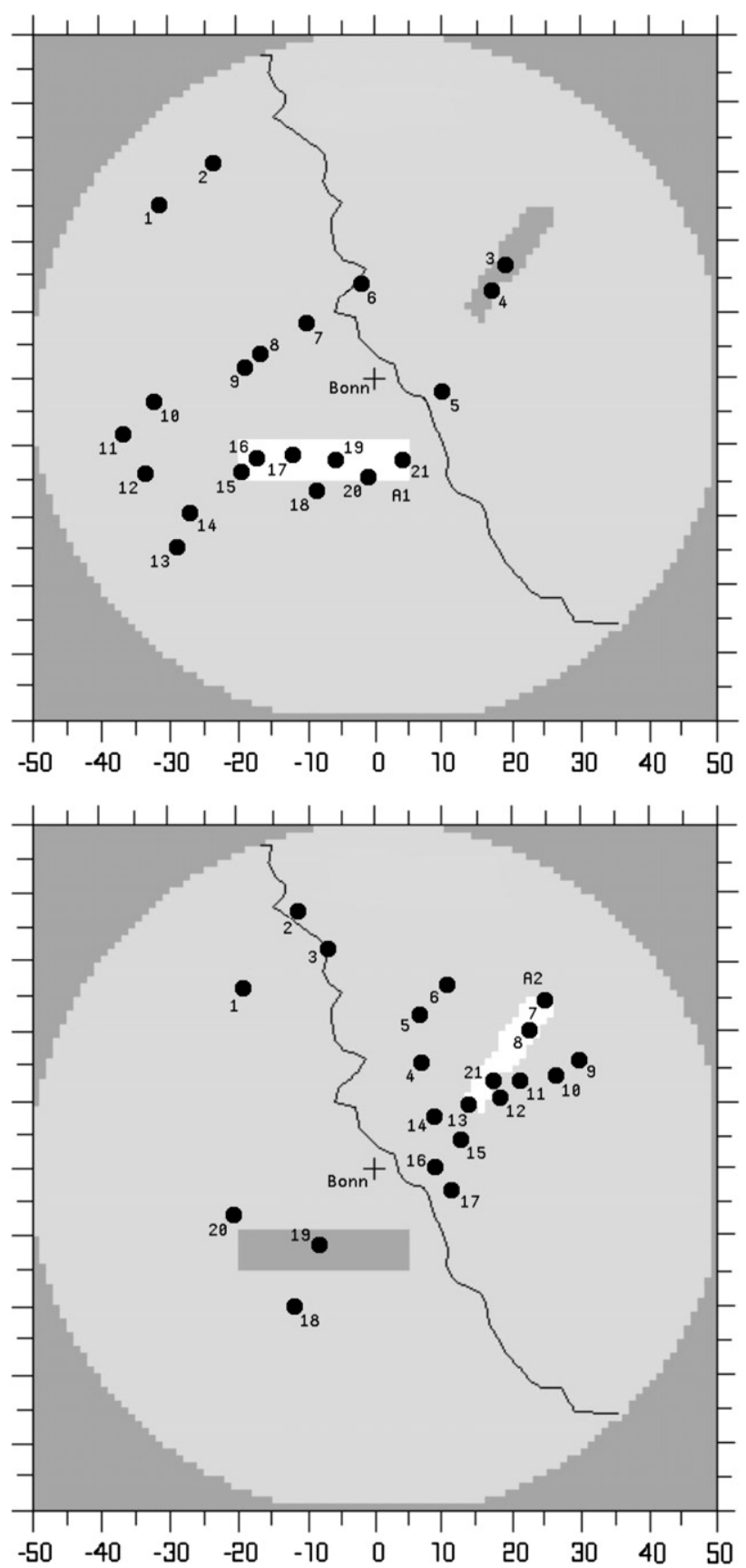

Figure 2 Top: Rain gauge cluster I, together with Ahr catchment area (A1, white) and Wahn catchment area (grey), bottom: rain gauge cluster II, together with Wahn catchment area (A2, white) and Ahr catchment area (grey).

SFB350 at the university of Bonn. The SFB rain gauges were maintained with high-frequency, and a quality control was performed including correlations between neighbouring stations and automated quality control using radar data. The number of available rain gauges is variable for different months, since some stations are rejected by the quality control checks.

Nearly continuous recordings of the radar and the rain gauges are available for the months June to September in the years 1998 and 1999, that is, a data set of eight months in total. The number of rain days varies between 5 and 13

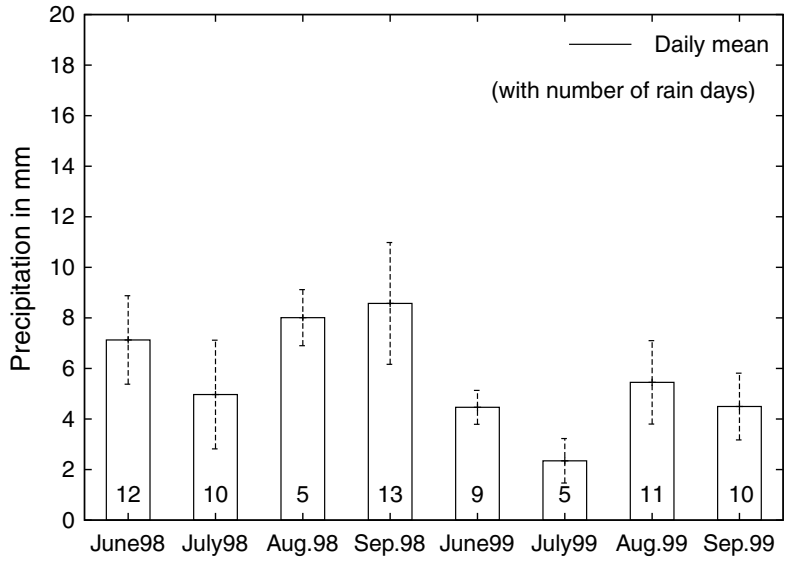

Figure 3 Mean precipitation sums with corresponding standard deviation, averaged over all rain days (the number of rain days is given in every box) and all available rain gauges.

for each month (Fig. 3). The mean daily precipitation sums related only to rain days is highest for August and September 1998. The monthly precipitation sums, averaged over all available rain gauges is highest for September 1998, which accounts also for the most rain days of all eight months. Thus, this month most significantly effects the results of this study. The standard deviation varies only slightly in between the eight months.

\section{Processing pipeline}

The standard preprocessing of the MIUB radar data uses an attenuation correction scheme, the elimination of clutter and the transformation from polar to Cartesian coordinates. In addition, the radar reflectivities are converted into rain rates, using a given $Z-R$ relationship. The choice of a suitable $Z-R$ relationship highly depends on the precipitation type. Differences are most pronounced for convective and stratiform precipitation events. Hence a statistical algorithm is performed to identify convective and stratiform precipitation events. In a first step, preprocessed radar reflectivities are converted into rain rates using a uniform $Z-R$ relationship suitable for stratiform precipitation events. If the precipitation event is classified as convective, the radar raw data are submitted to the preprocessing procedure a second time with a $Z-R$ relationship suitable for convective precipitation events. This is necessary because the attenuation correction depends on the choice of the $Z-R$ relationship. In the next step, a bias correction is applied to the obtained rain rates. Afterwards, an advection correction is performed before the radar rain rates are accumulated. Then, a modified statistical objective analysis scheme is used in order to adjust the radar precipitation to the rain gauge measurements. In a final step, areal precipitation sums are determined. The whole procedure sequence is shown in Fig. 4. In the following, the different steps are illustrated in more detail.

\section{Standard preprocessing of radar data}

Particularly in the case of heavy rainfall, attenuation must be taken into account. Thereby, the attenuation of the 


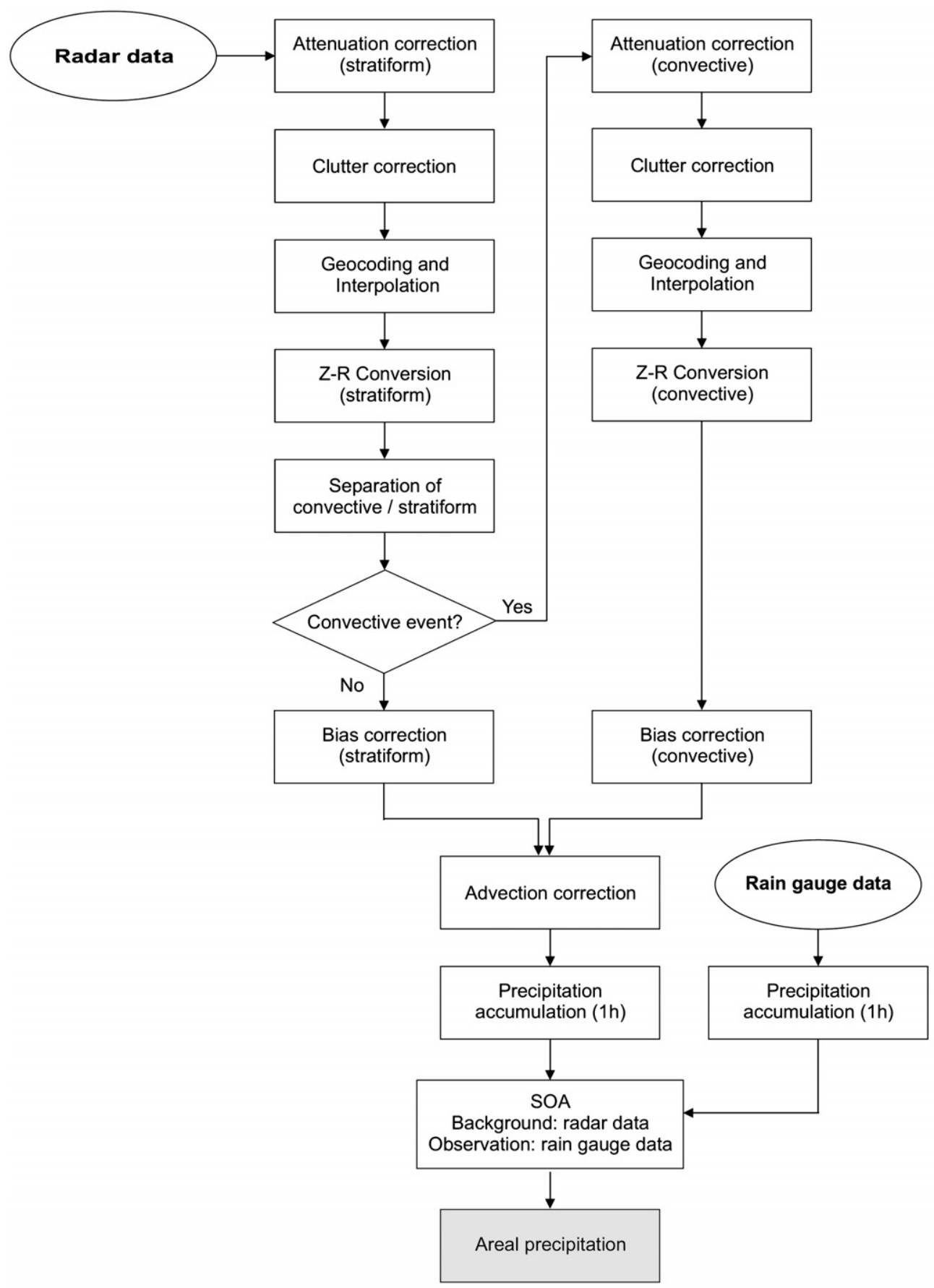

Figure 4 Flowchart of the real-time areal radar precipitation algorithm.

radar beam is usually parameterized by the rain rate. The attenuation coefficient $K_{d}(\mathrm{~dB} / \mathrm{km})$ is typically given by $K_{d}=\beta \cdot R^{\gamma}$ (Gunn and East, 1954). This way, the attenuation along a radar beam can be successively calculated. In this study, the parameters $\beta=0.0119$ and $\gamma=1.063$ by Eissing (1976) are used as they are best suited for the Bonn X-band radar (Kammer, 1991). This method for attenuation correction is the operational method for the X-band radar at Bonn. The dependence of attenuation correction algorithms on the drop size distribution has been studied in Delrieu et al. (1999) and on the radar calibration in Berne and Uij- lenhoet (2006). Although instabilities may occur during the successive correction, the method has proven to be stable for the data of the Bonn radar. Before a quantitative interpretation of the radar data is possible, false radar signals (clutter) must be eliminated. For this purpose, the radar reflectivities of rain-free days are combined into clutter maps, which are used to correct the actual radar data. The clutter maps are updated every month. Single radar beams with beam blocking effects are eliminated. In the next step, the polar coordinates of the raw data are transformed into Cartesian coordinates with a grid width of 
$1 \times 1 \mathrm{~km}^{2}$, in total $100 \times 100$ grid points. For this transformation, all measurement points within a radius of $500 \mathrm{~m}$ around a grid point are taken into account. The measurement points within this circle are weighted with a function that decreases exponentially with increasing distance to the grid point. Finally, Gauss-Krüger coordinates for every radar pixel are determined.

\section{Choice of $Z-R$ relationship}

The $Z-R$ relationship is generally formulated as $Z=a R^{b}$. The use of a uniform $Z-R$ relationship can lead to a severe over- or underestimation of the precipitation, since the rain drop size distribution varies highly with geographical localization, season, type and life cycle of the precipitation event. The difference can amount up to $800 \%$ of the real precipitation (Doelling et al., 1998). In order to reduce this error, it is a useful practice to distinguish between convective and stratiform rainfall events, because the drop size distributions of these precipitation types show the largest differences (Tokay and Short, 1996; Houze, 1993).

In the present study two $Z-R$ relationships are employed: Marshall et al. (1955) (M1955 in the following) with $a=200$ and $b=1.60$ for stratiform rainfall events, and Fujiwara (1965) with $a=450$ and $b=1.46$ for convective rainfall events. However, for real-time precipitation determination it is necessary to distinguish between convective and stratiform events automatically, based on the radar data only. Several radar-based classifications of convective and stratiform rainfall events have been proposed in the literature, e.g., using the intensity and sharpness of maxima of horizontal radar reflectivity patterns (Raghavan, 2003; Biggerstaff and Listemaa, 2000; Steiner et al., 1995), the bright band fraction (Chen and Uyeda, 2003), reflectivity areas and texture parameters (Beek et al., 2005; Walther and Bennartz, 2006).

In our study, a manual classification is performed at first in order to generate a reference data set. Thereby, the main interest lies in the clear identification of convective precipitation events. All other events (including mixed stratiform/convective cases) are summarized as stratiform rainfall events and will be treated with the $M 1955 Z-R$ relationship. The automatic stratiform/convective separation is performed by means of a spatial structure analysis. It is based on the assumption that the scale of convective precipitation cells is smaller than that of stratiform precipitation areas. Correlograms are computed for each azimuth scan, and are approximated by a function exponentially decreasing with distance. A correlogram establishes a statistical correlation between two observations and their distance $h$. Since the correlogram is computed from the field of radar-derived precipitation, it contains averaged information about the spatial structure of the precipitation cells. The general possibility to distinguish between convective and stratiform rainfall events by means of a spatial structure analysis is shown in Fig. 5, where mean correlograms for both precipitation types have been computed from a total of 2127 events. The automatic separation scheme uses the exponential coefficient of the correlogramm for an actual radar scan. A threshhold of -0.193 for the exponential

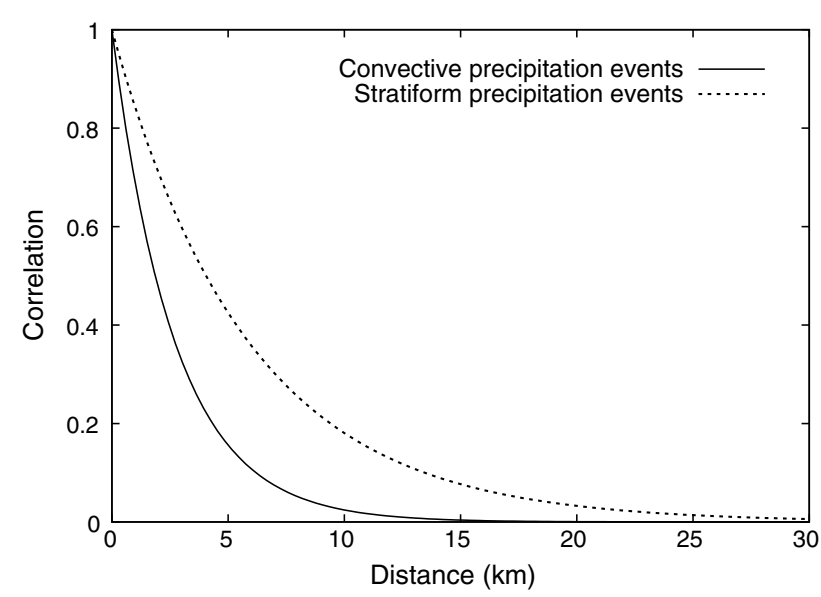

Figure 5 Mean correlograms for stratiform and convective precipitation events (manually classified azimuth scans).

coefficient was found to yield a suitable separation between convective and stratiform events. This threshhold was derived using the frequency distribution of the coefficients of all correlograms of manually classified events. For the threshhold of -0.193 the total error probability for the detection is about 24\% (Heuel, 2004).

\section{Bias correction}

In the next step, systematic errors due to over- or underestimation of the radar precipitation are eliminated from the radar data (see, e.g., Zawadzki, 1975). For this purpose, the radar precipitation is compared to rain gauge measurements of cluster I and II for a time series of eight months. The bias is computed by a temporal mean of all rain gauges with a correlation of at least 0.5 with the radar precipitation, based on daily precipitation sums. The actual correction of the radar data is performed using a mean correction factor $f$ for the whole period of eight months given by

$f=\frac{1}{K} \sum_{i=1}^{K} f_{i} \quad$ with $f_{i}=\sum_{j=1}^{N} P_{\mathrm{g}}^{j}\left(\boldsymbol{x}_{i}, \mathbf{y}_{i}\right) / \sum_{j=1}^{N} P_{\mathrm{r}}^{j}\left(\boldsymbol{x}_{i}, \mathbf{y}_{i}\right)$,

where $P_{r}^{j}$ is the radar precipitation and $P_{g}^{j}$ the rain gauge precipitation at time $j$ and grid point $\left(x_{i}, y_{i}\right), N$ the number of measurements and $K$ the number of rain gauges. Thereby, possible negative precipitation values are avoided. Actually, the bias correction corresponds to an adaption of the coefficient $a$ of the $Z-R$ relationship, resulting in values of $a=286$ and $a=307$ for the M1955 and Fujiwara (1965) Z$R$ relationships, respectively.

\section{Advection correction}

In order to generate hourly accumulation maps it is necessary to successively accumulate the rain rates of two consecutive radar scans. This is often done by a simple addition scheme that in principle only averages the corresponding values of the two input maps. However, it is more realistic to assume that in a certain time interval the precipitation event does not jump from one position to the next, but moves continuously and changes its intensity. Therefore, an advection 
correction scheme is used here that takes into account the movement of the precipitation patterns (see Handwerker, 2002 for the tracking of convective cells). For this purpose, the wind field is determined from the radar data. We use a modification of the cross correlation method proposed by Anagnostou and Krajewski (1999). The radar map is divided into $10 \times 10$ subdomains with a size of $10 \times 10$ pixels $(10 \mathrm{~km} \times 10 \mathrm{~km})$. For each of the 100 subdomains in the original scan the cross correlation with all subdomains of equal size within a certain square search area in the consecutive scan is computed. The search area is defined by the position of the original subdomain, shifted by the previous wind vector, and extended by $U$ pixels ( $U$ being 5 or 10 , see below) in each direction. Thus, areas in the consecutive scan that lie too far from the expected position of the new wind vector are eliminated at the beginning of the search procedure. At the boundary of the radar maps, only subdomains are considered, in which at least $75 \%$ of the pixels are located within the radar range. Subdomains, where the mean precipitation is below a threshold of $0.1 \mathrm{~mm}$, are also not taken into account. This way, up to $(2 U+1)^{2}$ different subdomains in the consecutive scan per subdomain in the original scan are checked. In the case of 5-min-scans, $U$ is set to 5 pixels, for the radar scans with a lower temporal resolution, $U$ is set to 10 pixels. Before the best correlated subdomain is chosen for the temporal interpolation, the computed correlation coefficients for each subdomain are weighted with two further parameters, the distance to the expected wind vector location and the ratio of the mean values of the two subdomains. For a detailed description of the applied procedure we refer to Heuel (2004). From the so-identified wind vectors for all subdomains finally one mean vector for the whole radar domain is determined. Along this mean wind vector the precipitation intensity is linearly interpolated between two successive radar scans. If a precipitation cell moves into or out of the radar range area, the rain rate of some pixels in one of the two considered radar scans is unknown. In these cases, the rain rate is assumed to remain constant. We decided to apply the advection correction after the bias adjustment in our operational scheme (Fig. 4), since the bias correction is an overall correction for possible long term radar calibration problems. However, for the sensitivity studies shown below all algorithm components can be applied separately.

\section{Statistical objective analysis}

The statistical objective analysis (SOA technique) uses observational data that are combined with a background field to generate an analysis field with a reduced expected error-variance. Pereira Fo and Crawford (1999) use temporally and spatially highly resolved radar data for the background field and a dense rain gauge network for the observation field. The analysed precipitation field is obtained by multiplying the bias-corrected radar pixels with the sum of the weighted differences between radar and rain gauge measurements. In this study we adjust and modify the SOA technique proposed by Pereira Fo and Crawford (1999) for real-time precipitation determination. For a detailed description of the mathematical derivation of the model equations we refer to Daley (1991) and Pereira Fo et al. (1998), see also Seo (1998).
The basic analysis equation of the SOA technique is

$P_{\mathrm{a}}\left(x_{i}, y_{i}\right)=P_{\mathrm{r}}\left(x_{i}, y_{i}\right)+\sum_{k=1}^{K} w_{i k}\left[P_{\mathrm{g}}\left(x_{k}, y_{k}\right)-P_{\mathrm{r}}\left(x_{k}, y_{k}\right)\right]$

where $P_{\mathrm{a}}\left(\boldsymbol{x}_{i}, \boldsymbol{y}_{i}\right)$ is the analysed precipitation and $P_{\mathrm{r}}\left(\boldsymbol{x}_{i}, \boldsymbol{y}_{i}\right)$ the radar precipitation at the grid point $\left(x_{i}, y_{i}\right), P_{\mathrm{g}}\left(x_{k}, y_{k}\right)$ is the rain gauge measurement and $P_{\mathrm{r}}\left(\boldsymbol{x}_{k}, \mathrm{y}_{k}\right)$ the radar measurement at the rain gauge position $\left(x_{k}, y_{k}\right)$. Furthermore, $w_{i k}$ is a to-be-determined a posteriori weight and $K$ the number of rain gauges. Minimizing the background error-variance leads to the following linear system for the weights:

$\sum_{l=1}^{K} w_{i l}\left(\rho_{k l}+\epsilon_{k l}^{2}\right)=\rho_{i k} \quad$ for $1 \leqslant k \leqslant K$,

with $\rho_{k l}$ being the background error cross correlation at the rain gauge positions $k$ and $l, \rho_{i k}$ the background error cross correlation between grid point $i$ and rain gauge position $k$, and $\epsilon_{k l}^{2}$ the normalized background error. Pereira Fo and Crawford (1999) show that the quality of the SOA estimation increases with increasing accumulation interval, using intervals between 15 and $120 \mathrm{~min}$. In this study, we use hourly rainfall sums (that is, data from rain gauges of the cluster I) for the SOA precipitation determination. Since the true rainfall is unknown, the background error must be estimated. Pereira Fo and Crawford (1999) or Michelson et al. (2000) use a single correlation function, which is computed as a temporal mean of numerous correlations. However, for real-time precipitation estimation a background error matrix is needed that is determined from the current weather situation. Therefore, a spatial mean is used instead of the temporal mean. This structure analysis is performed for every hourly accumulation map from which an actual correlation function is derived. In practice, the cross correlation $\rho_{k l}=\rho\left(\left(\boldsymbol{x}_{k}, \mathbf{y}_{k}\right),\left(\boldsymbol{x}_{l}, \mathbf{y}_{l}\right)\right)$ is replaced by a one-dimensional correlation function $\rho(h)$, which only depends on the distance $h$ between two pixels $\left(\boldsymbol{x}_{k}, \mathbf{y}_{k}\right)$ and $\left(\boldsymbol{x}_{l}, \boldsymbol{y}_{l}\right)$. The correlation function $\rho(h)$ is given by the mean cross correlation over all (equal sized) subdomains with distance $h$. For efficiency, one reference subdomain is defined in the center of the observation area and the cross correlation of the reference subdomain to every subdomain with distance $h$ in all horizontal, vertical and diagonal directions is computed. From these eight directions, the mean cross correlation function is determined, whereby the distance $h$ is evaluated up to a maximum of about $30 \mathrm{~km}$ (with a resolution of about $1 \mathrm{~km}$ ). A subdomain side length of 40 pixels was selected, since this choice resulted in the lowest RMS error (see Heuel, 2004). As for convective/stratiform separation, the correlation function for the structure analysis is approximated by a continuous model function of the form $\rho(h)=\mathrm{e}^{c h}$ that fulfils the necessary conditions for the applicability of the SOA scheme: $\rho(h)$ is positive monotonously decreasing and $\rho(0)=1$. For the SOA the correlation function is computed for the hourly values. For the normalized observation errors $\epsilon_{k k}$ a value of $0.1 \mathrm{~mm}$ for all rain gauges of the cluster $\mathrm{I}$ is assumed. Since the correlation functions are calculated every hour, an adaption to the actual situation is possible, which represents an improvement compared to procedures, which use predefined functions for the spatial 
interpolation of the rain gauge-calibrated radar data (e.g. RADOLAN, DWD, 2004).

\section{Case studies}

The performance of different algorithm components is now tested in various case studies. For validation, precipitation measurements of independent rain gauges are used.

\section{Dynamic selection of $Z-R$ relationships}

In order to test the manual separation, the radar reflectivities are uniformly converted with the $Z-R$ relationship for stratiform rainfall events as well as separately with the two $Z-R$ relationships for stratiform and convective rainfall events (see above). Then, the rainfall rates are accumulated to daily precipitation sums and compared with rain gauge measurements. In Fig. 6, the radar precipitation and the rain gauge precipitation for all five rain days in August 1998 (1 August 1998, 21-24 August 1998, encompassing four major rainfall events) is shown without and with the use of a dynamical $Z-R$ relationship. For this comparison, only the data of cluster II are used (daily measurements). If only the $M 1955 Z-R$ relationship is used, the daily mean precipitation (averaged over the 20 rain gauges of cluster II) is overestimated by $87 \%$, whereas the use of different $Z-R$ relationships for stratiform and convective precipitation events reduces the overestimation significantly (49\%). The correction works equally well for rain gauges measuring low as well as for rain gauges measuring high precipitation. A comparison of the automatic separation with the manual separation for the whole period of eight month shows that in 4359 of 7680 cases the manual results are detected (Table 1). Convective precipitation events, which are the main target of the identification, are detected with a success rate of $65 \%$ by the automatic separation.

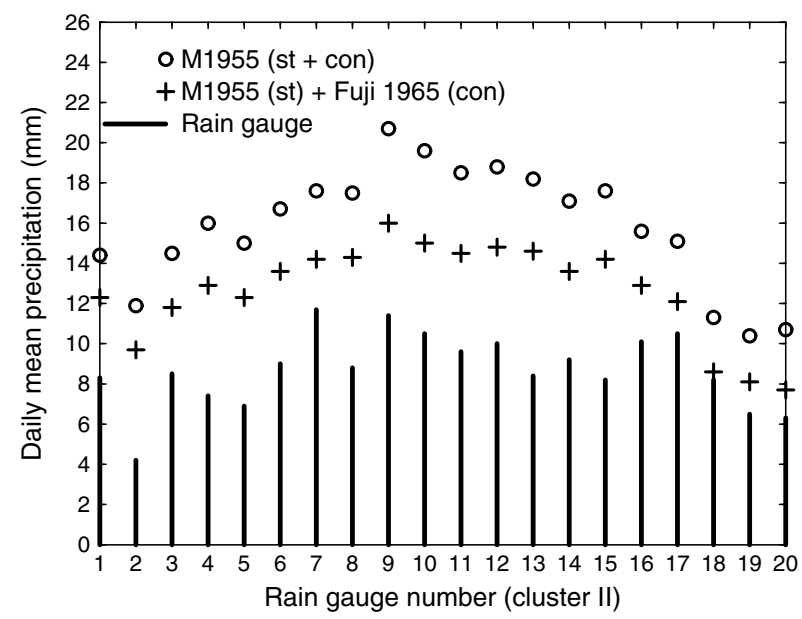

Figure 6 Comparison of rain gauge and radar precipitation, calculated with a uniform $Z-R$ relationship for stratiform (st) and convective (con) precipitation events (M1955, st + con) and with two manual separated $Z-R$ relationships (M1955, st + Fujiwara, 1965, con) for five days in August 1998 (daily mean precipitation, see text).
Table 1 Comparison between the manual and the automatic separation of convective and stratiform precipitation events

\begin{tabular}{lcll}
\hline Manual separation & \multicolumn{2}{l}{ Automatic separation } \\
\hline Stratiform & \multirow{2}{*}{5972 cases } & Stratiform & 3247 cases \\
& & Convective & 2725 cases \\
Convective & \multirow{2}{*}{1708 cases } & Convective & 1112 cases \\
& & Stratiform & 596 cases \\
\hline
\end{tabular}

\section{Advection correction}

The advection correction scheme leads to strong improvements for fast moving precipitation patterns in form of small convective cells or bands, which was investigated for numerous case studies (not shown). In these cases, a simple averaging without consideration of the advection leads to a pattern with two separated precipitation cells or bands. If a rain gauge is located, e.g. in the artificial gap that encloses the rainfall free zone, the advection correction results in a much better precipitation estimation since it produces a continuous precipitation pattern. An example for the impact of the advection correction is shown in Fig. 7 for a case study on 24 August 1998. The daily sum of radar precipitation without and with advection correction (AC) is shown for the positions of a subset of the rain gauges of cluster II. The impact of the AC varies largely ranging from a reduction by more than $5 \mathrm{~mm}$ to an increase of more than $5 \mathrm{~mm}$. Thus the impact can be large at individual stations, but it almost cancels out for areal averages or long term averages such as monthly means.

\section{SOA scheme}

The SOA scheme is applied to 1 - $h$ means of bias- and advection-corrected radar data. For the analysis, 15 rain gauges of the high-resolution cluster I are used ( 5 min data averaged to $1 \mathrm{~h}$ sums). The $1-\mathrm{h}$ SOA-corrected precipitation fields for the whole area covered by the radar are then averaged to daily sums. The data of rain gauge cluster II are not

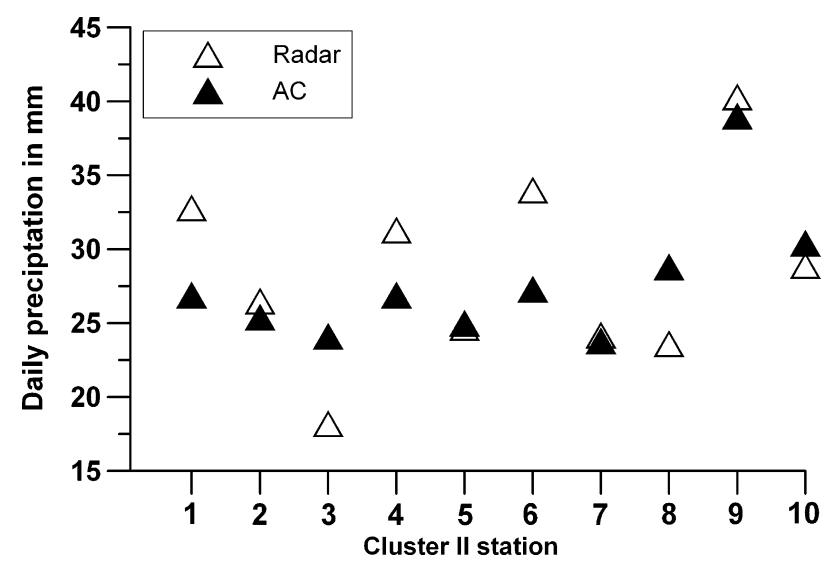

Figure 7 Comparison of precipitation sums for 24 August 1998 for radar and radar with advection correction (AC) at the positions of rain gauges $1-10$ of cluster II. 


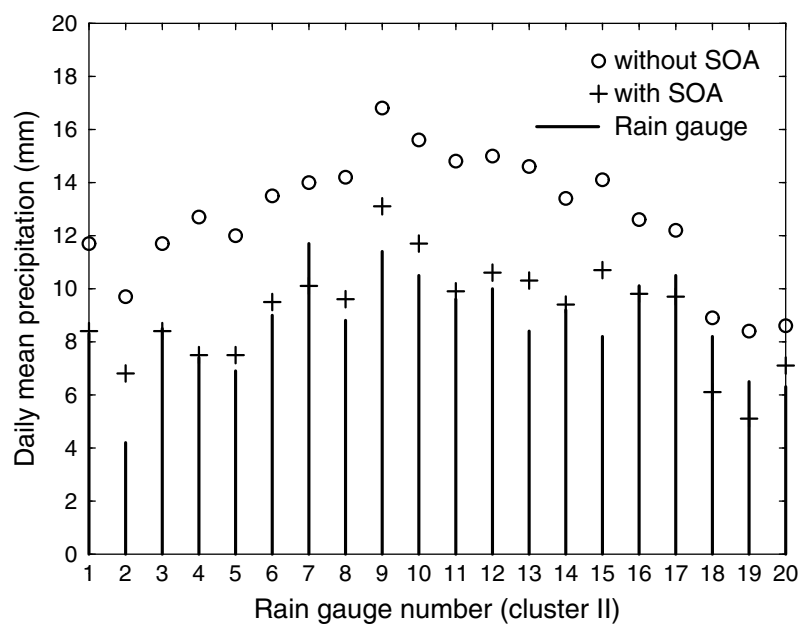

Figure 8 Comparison of rain gauge (cluster II) and radar precipitation, calculated before and after SOA analysis (with bias and advection correction) for five days in August 1998 (see text). Top: ratio of radar to rain gauge precipitation, bottom: daily mean precipitation.

used in the SOA, but are taken as an independent data set for the validation for the daily sums. In Fig. 8, the daily mean radar precipitation and the rain gauge precipitation of cluster II for the considered five days in August 1998 is shown before and after SOA. The SOA method reduces the ratio of radar to rain gauge precipitation (20 rain gauges of the independent rain gauge cluster II) from about 1.5 to nearly 1 . The daily mean precipitation after SOA agrees much better with the rain gauge measurements. For all rain gauges but for gauge \#18, the SOA scheme improves the accuracy of the radar precipitation. The average difference between radar precipitation and rain gauge measurements decreases through the application of the SOA method from about $4 \mathrm{~mm}$ to about $1 \mathrm{~mm}$ for this month. This shows that the effect of the SOA method is larger than the choice of the $Z-R$ relationship.

\section{Influence of rain gauge density}

A sensitivity study has been performed in order to assess the impact of the rain gauge density on the performance of the SOA scheme. For this purpose, the rain gauge density using the rain gauges of cluster I (used for the SOA) in the summer months August and September 1998 is decreased in several steps down to four gauges. The RMS differences, based on daily precipitation sums, are calculated using rain gauges of cluster II (Fig. 2), which are not used for the SOA.

In August 1998, the mean RMS difference without application of the SOA technique is $6.0 \mathrm{~mm}$. After the application of the SOA using only four rain gauges of the cluster I (mean distance about $30 \mathrm{~km}$ ) the RMS difference is reduced to $5.1 \mathrm{~mm}$. When 15 rain gauges are used, a further reduction to $4.8 \mathrm{~mm}$ is achieved. In September 1998, the radar precipitation measurement has a large bias in comparison to the rain gauge measurements. Without the usage of the SOA method the mean RMS difference is $21.1 \mathrm{~mm}$. After the application of the SOA technique a RMS difference of $8.6 \mathrm{~mm}$ can be achieved with only four rain gauges. The use of 15 rain gauges leads to a further improvement of only about $6 \%$. In both cases, the RMS difference decreases almost continuously with an increasing rain gauge density. In total, the SOA technique reacts stably to the elimination of individual rain gauges and can be efficiently used even with a low density rain gauge network for improving monthly means of regional precipitation.

\section{Validation study for the whole data set}

\section{Error quantification}

The radar rainfall estimation is evaluated using independent measurements of rain gauges from the two clusters. The calculated RMS differences are based on daily and hourly precipitation sums on a $1 \times 1 \mathrm{~km}^{2}$ radar grid. In addition, area-averaged radar and rain gauge precipitation sums are compared for two selected areas on a monthly basis. In both cases, the evaluation is performed separately for each algorithm of our processing pipeline as well as for various algorithm combinations in order to identify the contribution of each component and to find the optimum combination of components.

The quality of an interpolation scheme can be tested by comparing the measured and interpolated values at the same location. A necessary condition for the validity of such an analysis is that the measured values are not used for the interpolation. In our case, we want in particular to estimate the quality of a statistical objective analysis (SOA) scheme incorporating radar and ground measurements of precipitation. As mentioned above, the SOA method is based on hourly precipitation sums of the rain gauge cluster I. For validation on a daily basis, the rain gauges of cluster II can be used, which are completely independent from the SOA product. For validation on a hourly basis, we use the leave-one-outtechnique, that is the rain gauge of cluster I whose rainfall rate is used for the error evaluation is not included in the SOA method. This analysis is repeated for all rain gauges of cluster I available in each month. Thus, the RMS difference is computed by

$$
\mathrm{RMS}=\sqrt{\left(\sum_{j=1}^{N} K_{j}\right)^{-1} \sum_{j=1}^{N} \sum_{i=1}^{K_{j}}\left(P_{\mathrm{g}}^{j}\left(\boldsymbol{x}_{i}, \mathrm{y}_{i}\right)-P_{\mathrm{a}}^{j}\left(\boldsymbol{x}_{i}, \mathrm{y}_{i}\right)\right)^{2}}
$$

with $N$ being the number of days (either within a month or all 75 considered days), $K_{j}$ the number of rain gauges at the time $j$ (varying between 34 and 39 ), $P_{\mathrm{g}}^{j}$ the rain gauge precipitation at the time $j$ and the grid point $\left(\boldsymbol{x}_{i}, \boldsymbol{y}_{i}\right)$ and $P_{\mathrm{a}}^{j}$ the analysed precipitation at the time $j$ and the grid point $\left(x_{i}, y_{i}\right)$. For the computation of $P_{a}^{j}\left(x_{i}, y_{i}\right)$, the rain gauge at the grid point $\left(x_{i}, y_{i}\right)$ is not used for the analysis.

Additionally, hourly precipitation sums are considered instead of daily sums (thereby excluding cluster II), by replacing $N$ with the number of hours instead of days in Eq. (4).

\section{Individual analysis}

First, the quantitative impact of the various components of the algorithm (advection correction scheme, choice of $Z-R$ relationship, bias correction and SOA) on the error reduction for the radar precipitation estimation is examined. 
Fig. 3 gives an overview over the measured precipitation sums as an average of all rain gauges.

\section{Impact of advection correction}

The advection correction is performed by a spatio-temporal interpolation of the radar precipitation along an average wind vector computed from two subsequent radar scans. In a first step, the impact on the RMS difference (separately for each month) after the performance of the advection correction is assessed. In $57 \%$ of the comparisons an improvement is achieved by the application of the advection correction, but the impact is very small on a monthly basis (in the range of $-4 \%$ and $4 \%$, Fig. 9). The mean taken over all eight months and available rain gauges leads to a RMS difference of $11.6 \mathrm{~mm}$ without and to $11.5 \mathrm{~mm}$ with application of the advection correction. That means an only small improvement of about $1 \%$. However, the advection correction leads to more significant improvements over short time intervals based on hourly precipitation sums (Fig. 10). Here, its monthly influence varies between about $-1 \%$ and about $10 \%$.

\section{Impact of $Z-R$ relationship}

We use two separate $Z-R$ relationships, one for stratiform and one for convective precipitation events. The type of event is detected either automatically by a structural analysis of the rain field or manually.

A comparison of the RMS differences, based on daily precipitation sums, shows an error reduction in about $78 \%$ of the comparisons for the manual separation. The mean taken over all eight months and available rain gauges leads to a RMS difference of $11.5 \mathrm{~mm}$ without and to $6.9 \mathrm{~mm}$ with application of the manual separation. This means an average error reduction of about $40 \%$.

The application of the automatic separation leads to an improvement in about $73 \%$ of the comparisons for the monthly means. Averaged over all months and rain gauges, the automatic separation method results in an error reduction of circa $9 \%$, which means a RMS difference of $10.5 \mathrm{~mm}$. One reason for this decrease in improvement compared to

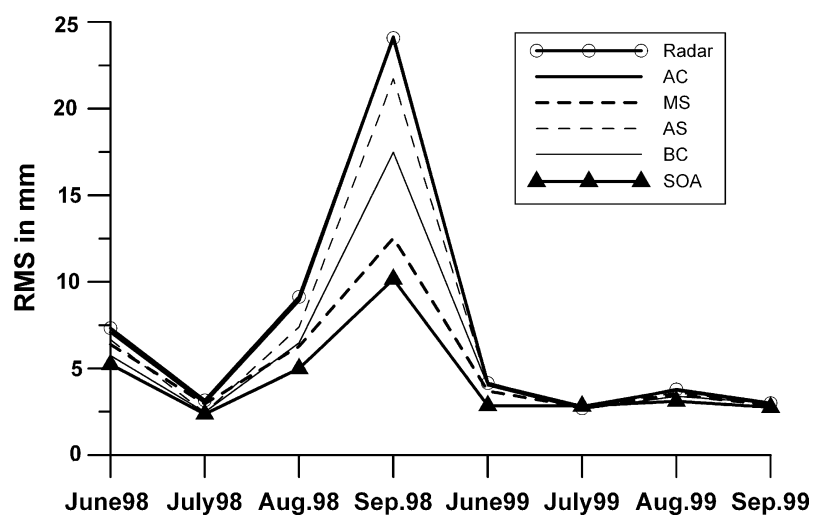

Figure 9 RMS differences between rain gauge and radar precipitation (rain gauge cluster I and II), based on daily precipitation sums, calculated for the uncorrected radar data (Radar), with advection correction (AC), with manual separation (MS), with automatic separation (AS), with bias correction (BC) and with SOA.

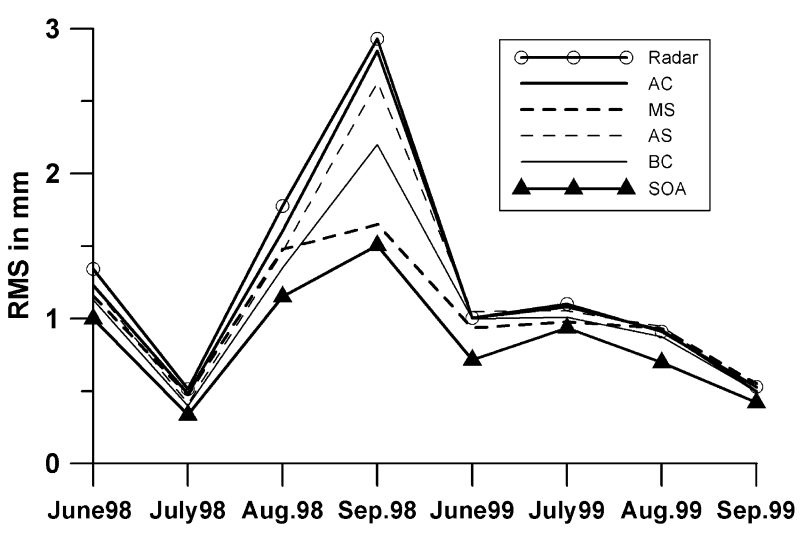

Figure 10 RMS differences between rain gauge and radar precipitation (rain gauge cluster I), based on hourly precipitation sums (without zero values), calculated for the uncorrected radar data (Radar), with advection correction (AC), with manual separation (MS), with automatic separation (AS), with bias correction (BC) and with SOA.

the manual method is that some very large convective precipitation systems are detected as stratiform events by the automatic separation method.

Fig. 9 shows the statistical results separately for each month. The improvement gained by the manual separation varies between about $2 \%$ and $50 \%$ and leads in no case to a deterioration of the RMS difference. The automatic method can lead to an improvement of at most $20 \%$ and also does not lead to an increase of the RMS difference. Similar results are obtained using the RMS differences based on hourly precipitation sums (Fig. 10).

\section{Impact of bias correction}

The bias correction alters the radar precipitation with a correction factor determined from the ratio of the mean rain gauge and radar precipitations for the complete data set. The bias correction leads to an improvement in $75 \%$ of the comparisons for the RMS differences based on daily precipitation sums. Fig. 9 shows the statistical results separately for each month. The improvement gained by the bias correction varies between about $-2 \%$ and $28 \%$. The mean taken over all eight months and available rain gauges leads to a RMS difference of $11.5 \mathrm{~mm}$ without and to $8.7 \mathrm{~mm}$ with application of the bias correction. That means an error reduction of about $24 \%$. The comparison of the RMS differences based on hourly precipitation sums of rain gauge cluster I shows similar results (Fig. 10). Here, the average error reduction is about $18 \%$.

\section{Impact of SOA}

The SOA method corrects the radar precipitation using rain gauge measurements minimizing the expected error variance. In $78 \%$ of the comparisons an improvement of the RMS differences, based on daily precipitation sums, is achieved by the application of the SOA technique. Here, the rain gauges of the cluster I are used in the leave-oneout validation. The mean taken over all eight months and all available rain gauges leads to a RMS difference of $11.5 \mathrm{~mm}$ without and to $6.0 \mathrm{~mm}$ with application of the SOA technique. That means an error reduction of about 
$48 \%$. Based on hourly precipitation sums (calculated only with rain gauges of cluster I), the RMS difference decreases in circa $75 \%$ of the comparisons. A distinction between convective and stratiform precipitation events in this statistic shows that the SOA technique leads to an improvement for circa $75 \%$ of the stratiform events. For the convective precipitation events an improvement for circa $78 \%$ of the events is achieved. This means that the SOA technique leads in 3/4 of the cases to an error reduction, independent of the precipitation type.

Furthermore, the RMS differences are considered separately for all eight months, based on daily precipitation events (Fig. 9). Here, considerable differences can be seen in the efficiency of the SOA technique for individual months. Especially, in September 1998 a very high error reduction is shown. For July to September 1999 the correction of all algorithm components is small for the monthly means of daily precipitation sums. The impact of the SOA is larger for the RMS differences based on hourly precipitation sums (Fig. 10), where an improvement by the SOA component can be seen for all months. Thus the SOA is beneficial particularly for single rain events. Since our whole processing pipeline is based on radar and rain gauges only, it has the potential to be applied in real time e.g. for flash flood forecasting provided that the rain gauge data is available in real time as well.

\section{Interaction of all components}

Now, the interaction of the individual algorithms is analysed. Hereby, not all possible combinations (with/without advection correction, with/without bias correction, with/ without SOA technique, with/without manual/automatic separation, corresponding to 24 possible combinations), but only a selection of combinations is shown, which may be of practical use. The evaluation is based on an extensive RMS error study by means of point-to-point (radar-rain rauge) comparisons as well as on comparisons of areal precipitation sums for two selected catchment areas within the radar range.

\section{Point-to-point comparisons}

First, we consider point-to-point comparisons similar to the previous chapter. Fig. 11 shows the RMS differences for 14 different combinations of radar processing algorithms, based on daily precipitation sums. The following conclusions can be drawn:

- the influence of the advection correction is small for the monthly means, which has already been observed in the individual study,

- the separation between stratiform and convective precipitation events has a large influence on the error reduction, if the bias correction is not performed; otherwise its influence is smaller,

- without bias correction the automatic separation is not as efficient as the manual separation,

- the manual separation leads to an error reduction of about $41 \%$ even without application of bias correction and SOA technique; this shows how important the choice of a suitable $Z-R$ relationship is,

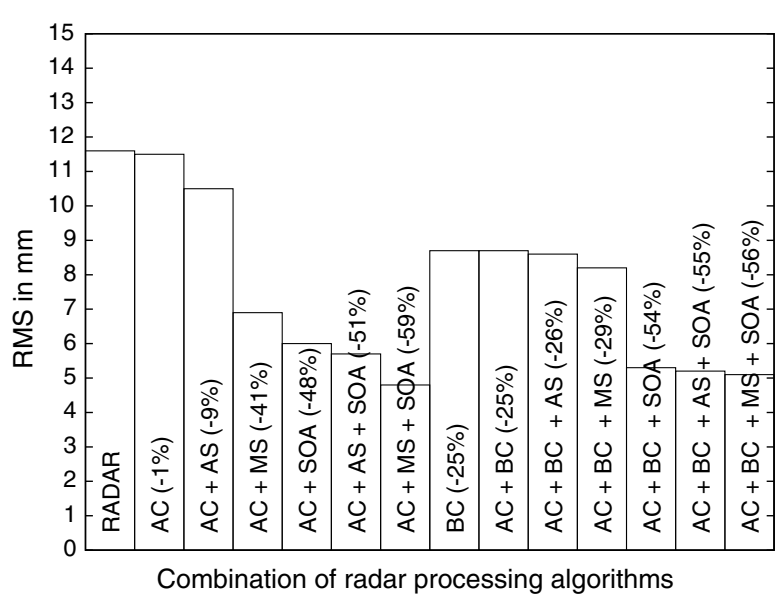

Figure 11 RMS differences between rain gauge and radar precipitation (rain gauge cluster I and II) for different algorithm combinations, calculated for a period of eight months, based on daily precipitation sums (AC, advection correction; AS, automatic separation; $M S$, manual separation; $B C$, bias correction).

- the bias correction alone leads to an error reduction of about 25\%; however, without the SOA technique the error cannot be reduced further,

- the SOA technique leads to an error reduction of about $55 \%$; the impact of the bias correction is small, if the SOA is performed.

The optimal combination in this study consists of the simultaneous employment of SOA technique and manual separation. For an automatic real-time estimation of precipitation the combination of SOA technique, advection correction, bias correction and automatic separation is recommended. Improvements of the automatic separation may be achieved using larger radar scan areas than the $50 \mathrm{~km}$ radius of our study.

\section{Areal comparisons}

For hydrological applications, the accurate knowledge of areal precipitation sums is very important. In the following, areal precipitation sums are examined for two small catchment areas. Thereby, only monthly precipitation sums are considered. For our study we select two catchment areas, one of them located within the rain gauge cluster I, the other within rain gauge cluster II. In rain gauge cluster I, a rectangular area $\left(\mathrm{A} 1,290 \mathrm{~km}^{2}\right)$ was defined that about represents approximately the catchment area of the Ahr river. In rain gauge cluster II the catchment area of the Wahn river (A2, $70 \mathrm{~km}^{2}$ ) was chosen (Fig. 2). The Wahn catchment area is very important for local water management purposes, since the Wahn river dam is the main water reservoir for the city of Bonn.

For an areal comparison of radar and rain gauge measurements, the point rain gauge measurement have to be interpolated over the considered area (compare Stellman et al., 2001). Such a comparison has the disadvantage that the quality of the comparison data is itself dependent on the quality of the used interpolation method. However, we assume that within a relative dense rain gauge network interpolated rain gauge precipitation by ordinary Kriging 


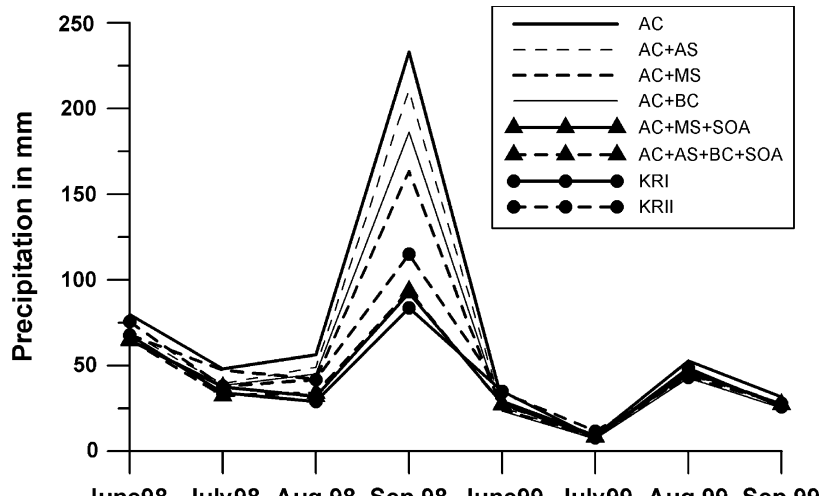

June98 July98 Aug.98 Sep.98 June99 July99 Aug.99 Sep.99

Figure 12 Mean areal radar and rain gauge precipitation (monthly precipitation sums in $\mathrm{mm}$ ) in area $\mathrm{A} 1$, calculated with different radar data processing algorithm combinations (AC, advection correction; AS, automatic separation; MS, manual separation; BC, bias correction; KRI, Kriging interpolation, based on rain gauge cluster I; KRII, Kriging interpolation, based on rain gauge cluster II).

(denoted by KRI for cluster I and KRII for cluster II) can be used as a comparison data set for the evaluation study for limited areas like $A 1$ and $A 2$. The Kriging variogram was estimated in $5 \mathrm{~km}$ bins for a maximum distance of $40 \mathrm{~km}$ using a spherical variogram function (for details see Heuel, 2004). An interpretation of the results must take into consideration that only the areal means for $\mathrm{A} 2$ computed by rain gauge cluster II are independent from the analysed radar precipitation, since the rain gauges of cluster I (area A1) are also used for the SOA technique. Thus, a second areal mean was computed using the data of rain gauges of the independent cluster II for area A1 (KRII).

Fig. 12 shows the results for the area $A 1$. The uncorrected radar data is not shown, since it is almost identical to AC. The application of the bias correction generally improves the results, additional improvement can be seen for the combination with the use of dynamic $Z-R$ relationships and the SOA technique (taking KRI values as reference). When the SOA is applied, the bias correction has only minor influence. In particular, highly overestimated precipitation sums by the radar (as, e.g., in September 1998) are reduced to the rain gauge precipitation level by SOA. Because of the low density of rain gauges of the operational DWD network (cluster II) in the A1 area, KRII values show considerable deviations from the KRI precipitation (generally an overestimation).

Fig. 13 shows the analysis results for the area A2 (where KRII is the reference). Largest differences between the SOA and the KRII can be seen for July 1998. In this month, the SOA leads to a slight underestimation of the precipitation sum. But, in general, the application of the SOA technique yields the best results compared to the ground truth measurements of rain gauge cluster II.

Overall it can be concluded that the application of the SOA technique together with the dynamical use of the $Z$ $R$ relationship for convective and stratiform precipitation events leads to a good approximation of the rain gauge precipitation and thus to a significantly better areal precipitation quantification from radar data. For the small

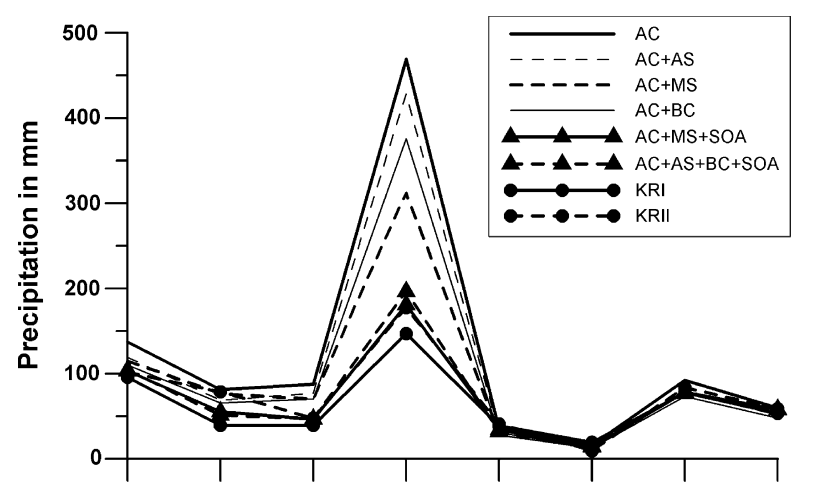

June98 July98 Aug.98 Sep.98 June99 July99 Aug.99 Sep.99

Figure 13 Mean areal radar and rain gauge precipitation (monthly precipitation sums in $\mathrm{mm}$ ) in area $\mathrm{A} 2$, calculated with different radar data processing algorithm combinations (AC, advection correction; AS, automatic separation; MS, manual separation; BC, bias correction; KRI, Kriging interpolation, based on rain gauge cluster I; KRII, Kriging interpolation, based on rain gauge cluster II).

catchment areas considered in this section, Kriging with a dense rain gauge network yields also good results for the monthly areal precipitation sums. But, as dicussed above, the impact of the SOA technique as well as the advection correction increases when the time scale is reduced.

\section{Summary and conclusions}

In this study, various procedures for the determination of radar precipitation are modified and adapted for a scheme of real-time areal precipitation quantification. In particular, the scheme includes a bias correction, an advection correction, a separation of convective and stratiform precipitation events, and a SOA technique.

The bias correction is a formal prerequisite for the application of the SOA technique. Actually, it corresponds to a correction of the factor $a$ of the $Z-R$ relationship. The advection correction interpolates the rain intensity spatiotemporally and thus reduces errors arising from sampling errors of radar scans with low temporal resolution. The (manual and) automatic separation of convective and stratiform rainfall events helps to prevent an over- or underestimation of the precipitation from an inappropriate choice of the Z$R$ relationship. Finally, the SOA scheme corrects the precipitation field with the help of ground measurements and leads to a significant improvement of the quality of the radar precipitation. When the SOA method is applied, the bias correction has almost no influence. Therefore, the SOA can also be used without bias correction, and can be used as a real-time precipitation determination. The efficiency of the procedures was illustrated for examples, and an error analysis was performed for eight months of data with all available radar and rain gauge measurements. The impact of the individual components as well as their interaction with each other was analysed.

The advection correction has large impact for single precipitation events. Averaged over longer time intervals, the advection correction does not contribute much to the error reduction. The bias correction is performed in order 
to eliminate systematic errors of the radar data by comparing radar precipitation with rain gauge measurements over a long period. In contrast to other studies (e.g. Stellman et al., 2001) we apply the same bias correction for the whole data set and not on a hourly or daily basis. Furthermore, the bias correction does not allow for local spatial corrections. Nevertheless, an error reduction of up to $28 \%$ is achieved.

The separation of convective and stratiform precipitation events enables an adjustment of the $Z-R$ relationship to the actual precipitation type. This shows a considerable error reduction in almost all cases. By the manually performed separation method a correction of up to $48 \%$ could be achieved. The automatic separation between convective and stratiform precipitation events based on a structure analysis of the radar data achieves an error reduction of up to $20 \%$.

The SOA technique enables a local correction of the radar measurements by using rain gauge data, which leads to an immense improvement of the quality of the radar precipitation quantification. Thereby, the original structure of the radar rainfall distribution is preserved, while the precipitation sums are adjusted to the ground truth. The SOA technique is especially suitable if the scale of the precipitation events is large compared to the density of the rain gauge network. Locally limited convective rainfall cells at large distances to rain gauges may lead to errors in the SOA. For real-time adjustment, the original SOA technique (Daley, 1991; Pereira Fo et al., 1998; Pereira Fo and Crawford, 1999) has been modified. Thus, good results are achieved also for short time intervals. Together with the advection and the bias correction, an error reduction of about $54 \%$ is achieved.

The best overall results are gained by a combination of the advection correction, the manual convective/stratiform separation method and the SOA technique. For monthly means, an average error reduction of about $59 \%$ was achieved. Hydrological applications, e.g., flood forecasting, highly depend on an accurate precipitation quantification of at least 10\% (Joss et al., 1998). The performance of the complete processing pipeline leads to a considerable error reduction of the radar precipitation estimation. Our results show that our multicomponental scheme represents one step towards achieving the desired accuracy.

\section{Acknowledgements}

This research was supported by the 'Sonderforschungsbereich (SFB) 350' of the DFG. The authors thank the DWD and the Erftkreisverband for the supply with rain gauge data and the remote sensing group of the MIUB (especially D. Meetschen and T. Grimbacher) for the supply with radar data as well as with the corresponding preprocessing software.

\section{References}

Anagnostou, E.N., Krajewski, W.F., 1999. Real-time radar rainfall estimation. Part I: Algorithm formulation. J. Atmos. Ocean. Technol. 16, 189-197.
Beek, C.Z. van de, Uijlenhoet, R., Holleman, I., 2005. Spatial classification of precipitation from operational radar data. In: Proceedings of the 32nd Conference on Radar Meteorology 2005, Albuquerque, AMS.

Berne, A., Uijlenhoet, R., 2006. Quantitative analysis of X-band weather radar attenuation correction accuracy. Nat. Hazards Earth Syst. Sci. 6, 419-425.

Biggerstaff, M.I., Listemaa, S.A., 2000. An improved scheme for convective stratiform echo classification using radar reflectivity. J. Appl. Meteorol. 39, 2129-2150.

Chen, J., Uyeda, H., 2003. A method using radar reflectivity data for the objective classification of precipitation during the Baiu season. J. Meteorol. Soc. Jpn. 81 (2), 229-249.

Daley, R., 1991. Atmospheric Data Analysis. Cambridge University Press, Cambridge, 457pp.

Delrieu, G., Hucke, L., Creutin, J.D., 1999. Attenuation in rain for $X$ - and C-band weather radar systems: sensitivity with respect to the drop size distribution. J. Appl. Meteorol. 38, 57-68.

Doelling, I.G., Joss, J., Riedl, J., 1998. Systematic variations of Z$R$-relationships from drop size distributions measured in northern Germany during seven years. Atmos. Res. 47-48, 635-649.

DWD, 2004. Final report RADOLAN. Online. Available at: <http:// www.dwd.de/de/wir/Geschaeftsfelder/Hydrometeorologie/ a_href_pages/RADOLAN>.

Eissing, R., 1976. Streuung und Dämpfung elektromagnetischer Wellen an Niederschlägen in $\mathrm{cm} / \mathrm{mm}$ - Wellenlängenbereichen. Berichte des Instituts für Radarmeteorologie und Maritime Meteorologie 33, Universität Hamburg.

Fujiwara, M., 1965. Raindrop-size distribution from individual storms. J. Atmos. Sci. 22, 585-591.

Fulton, R.A., Breidenbach, J.P., Seo, D., Miller, D.A., O'Bannon, T., 1998. The WSR-88D rainfall algorithm. Weather Forecast. 13, 377-395.

Fulton, R.A., 1999. Sensitivity of WSR-88D rainfall estimates to the rain-rate threshold and rain gauge adjustment: a flash flood case study. Weather Forecast. 14, 604-624.

Gunn, K., East, T., 1954. The microwave properties of precipitation particles. Quart. J. Roy. Meteorol. Soc. 80, 522-545.

Handwerker, J., 2002. Cell tracking with TRACE3D - a new algorithm. Atmos. Res. 61, 15-34.

Heuel, E., 2004. Quantitative Niederschlagsbestimmung aus Radardaten. Ein Vergleich von unterschiedlichen Verfahren unter Einbeziehung der Statistischen Objektiven Analyse. Dissertation am Meteorologischen Institut der Universität Bonn, 162pp.

Houze, R.A., 1993. Cloud Dynamics. Academic Press, San Diego, 570pp.

Joss, J., Waldvogel, A., 1990. Precipitation measurement and hydrology. In: Radar in Meteorology. American Meteorological Society, Boston, pp. 577-606.

Joss, J., Meischner, P., Hagen, M., Riedl, J., Weigl, E., 1998. Quantitative Niederschlagsmessung. In: Annalen der Meteorologie 38, 101-110, Herbstschule Radarmeteorologie vom 2.-6. November 1998 in Oberpfaffenhofen, Selbstverlag des Deutschen Wetterdienstes, Offenbach/Main.

Kammer, A., 1991. A low cost X-band radar system designed for the use in urban hydrology. In: Preprints, 25th Conference on Radar Meteorology. American Meteorological Society, Paris.

Marshall, J.S., Hitschfeld, W., Gunn, K.L.S., 1955. Advances in radar weather. Adv. Geophys. 2, 1-56.

Matsoukas, C., Islam, S., Kothari, R., 1999. Fusion of radar and rain gage measurements for an accurate estimation of rainfall. J. Geophys. Res. 104 (D24), 437-450.

Michelson, D.B., Foltescu, V.L., Häggmark, L., Lindgren, B., 2000. MESAN Mesoscale analysis precipitation. Meteorol. Z. 9, 85-96.

Pereira Fo, A.J., Crawford, K.C., Hartzell, C.L., 1998. Improving WSR-88D hourly rainfall estimates. Weather Forecast. 13, 10161028. 
Pereira Fo, A.J., Crawford, K.C., 1999. Mesoscale precipitation fields. Part I: Statistical analysis and hydrologic response. J. Appl. Meteorol. 38, 82-101.

Raghavan, S., 2003. Radar Meteorology. Kluwer Academic Publishers, Dordrecht.

Reudenbach, C., Heinemann, G., Heuel, E., Bendix, J., Winiger, M., 2001. Investigation of summertime convective rainfall in Western Europe based on a synergy of remote sensing data and numerical models. Meteorol. Atmos. Phys. 76, 23-41.

Seo, D.J., 1998. Real-time estimation of rainfall fields using radar rainfall and rain gauge data. J. Hydrol. 208, 37-52.

Steiner, M., Houze, R.A., Yuter, S.E., 1995. Climatological characterization of three-dimensional storm structure from opera- tional radar and rain gauge data. J. Appl. Meteorol. 34, 19782007.

Stellman, K.M., Fuelberg, H.E., Garza, R., Mullusky, M., 2001. An examination of radar and rain gauge-derived mean areal precipitation over Georgia watersheds. Weather Forecast. 16, 133-144.

Tokay, A., Short, D.A., 1996. Evidence from tropical raindrop spectra of the origin of rain from stratiform versus convective clouds. J. Appl. Meteorol. 35, 355-371.

Walther, A., Bennartz, R., 2006. Radar-based precipitation type analysis in the Baltic area. Tellus 58A, 331-343.

Zawadzki, I.I., 1975. On radar-raingauge comparison. J. Appl. Meteorol. 14, 1430-1436. 